\title{
ON $\theta$-CONGRUENT NUMBERS ON REAL QUADRATIC NUMBER FIELDS
}

\author{
Ali S. Janfada and SAJad SAlami
}

\begin{abstract}
Let $\mathbf{K}=\mathbf{Q}(\sqrt{m})$ be a real quadratic number field, where $m>1$ is a squarefree integer. Suppose that $0<\theta<\pi$ has rational cosine, say $\cos (\theta)=s / r$ with $0<|s|<r$ and $\operatorname{gcd}(r, s)=1$. A positive integer $n$ is called a $(\mathbf{K}, \theta)$-congruent number if there is a triangle, called the $(\mathbf{K}, \theta, n)$-triangles, with sides in $\mathbf{K}$ having $\theta$ as an angle and $n \alpha_{\theta}$ as area, where $\alpha_{\theta}=\sqrt{r^{2}-s^{2}}$. Consider the $(\mathbf{K}, \theta)$-congruent number elliptic curve $E_{n, \theta}: y^{2}=x(x+(r+s) n)(x-(r-s) n)$ defined over $\mathbf{K}$. Denote the squarefree part of positive integer $t$ by $\operatorname{sqf}(t)$. In this work, it is proved that if $m \neq \operatorname{sqf}(2 r(r-s))$ and $m n \neq 2,3,6$, then $n$ is a $(\mathbf{K}, \theta)$-congruent number if and only if the Mordell-Weil group $E_{n, \theta}(\mathbf{K})$ has positive rank, and all of the $(\mathbf{K}, \theta, n)$-triangles are classified in four types.
\end{abstract}

\section{Introduction}

A positive integer $n$ is called a congruent number if it is the area of a right triangle with rational sides. Finding all congruent numbers is one of the classical problems in the modern number theory. We cite [8] for an exposition of the congruent number problem, and [4] to see the first study of $\theta$-congruent numbers as a generalization of the classic one. Let $0<\theta<\pi$ has rational $\operatorname{cosine} \cos (\theta)=$ $s / r$ with $0<|s|<r$ and $\operatorname{gcd}(r, s)=1$. Let $(U, V, W)_{\theta}$ denote a triangle with an angle $\theta$ between sides $U$ and $V$. A positive integer $n$ is called a $\theta$-congruent number if there exists a triangle $(U, V, W)_{\theta}$ with sides in $\mathbf{Q}$ having area $n \alpha_{\theta}$, where $\alpha_{\theta}=\sqrt{r^{2}-s^{2}}$. In other words, $n$ is a $\theta$-congruent number if it satisfies

$$
2 r n=U V, \quad W^{2}=U^{2}+V^{2}-\frac{2 s}{r} U V .
$$

An ordinary congruent number is nothing but a $\pi / 2$-congruent number. Clearly, if $n$ is a $\theta$-congruent number, then so is $n t^{2}$, for any positive integer $t$. We shall concentrate on squarefree numbers whenever $\theta$-congruent numbers concerned. Let

$$
E_{n, \theta}: y^{2}=x(x+(r+s) n)(x-(r-s) n)
$$

2010 Mathematics Subject Classification. Primary 11G05, Secondary 14H52.

Key words and phrases. $\theta$-congruent number, elliptic curve, real quadratic number field. Received August 15, 2014; revised November 11, 2014. 
be the $\theta$-congruent number elliptic curve, where $r$ and $s$ are as above. Theorem 2.4 gives an important connection between $\theta$-congruent numbers and the MordellWeil group $E_{n, \theta}(\mathbf{Q})$. For more information and recent results about $\theta$-congruent numbers see $[5,3,14]$.

The notion $\theta$-congruent number, which is defined over $\mathbf{Q}$, can be extended in a natural way over real quadratic number fields $\mathbf{K}$. In this case, we refer to $n$ as a $(\mathbf{K}, \theta)$-congruent number and to the triangle $(U, V, W)_{\theta}$ as a $(\mathbf{K}, \theta, n)$-triangle. When $n$ is not a $\theta$-congruent number over $\mathbf{Q}$, a question proposed naturally: $I s$ $n$ a $(\mathbf{K}, \theta)$-congruent number for some real quadratic number field $\mathbf{K}$ ? Tada [13] answered this question in the case $\theta=\pi / 2$, by studying the structure of the K-rational points on the elliptic curve $E_{n, \pi / 2}: y^{2}=x\left(x^{2}-n^{2}\right)$. In this paper, we answer the above question for any $0<\theta<\pi$ and classify all $(\mathbf{K}, \theta, n)$-triangles. Through the paper we shall consider $\mathbf{K}=\mathbf{Q}(\sqrt{m})$ to be a real quadratic field, where $m>1$ is squarefree. We denote the squarefree part of any positive integer $N$ by $\operatorname{sqf}(N)$. The main results of this paper are the following theorems.

THEOREM 1.1. Let $n$ be a positive squarefree integer with $\operatorname{gcd}(m, n)=1$ such that $m n \neq 2,3,6$ and $m \neq \operatorname{sqf}(2 r(r-s))$, where $m, r, s$ are as before. Then $n$ is $a(\mathbf{K}, \theta)$-congruent number if and only if $\operatorname{rank}\left(E_{n, \theta}(\mathbf{K})\right)>0$. Moreover, $n$ is a $(\mathbf{K}, \theta)$-congruent number if and only if either $n$ or $m n$ is a $\theta$-congruent number over $\mathbf{Q}$.

Theorem 1.1 is an extension of Part (2) of Theorem 2.4 in the following. Note that the non-equality conditions for $m n$ and $m$ in Theorem 1.1 are necessary. For a counterexample, when $n=1$ and $\theta=2 \pi / 3$, we have $r=2, s=-1$, $\alpha_{\theta}=\sqrt{3}$. Now taking $m=3=\operatorname{sqf}(2 r(r-s))$, there is a $(\mathbf{Q}(\sqrt{3}), \theta, 1)$-triangle with sides $(2,2,2 \sqrt{3})$ and area $\sqrt{3}$ but using Theorem $2.1, \operatorname{rank}\left(E_{1, \theta}(\mathbf{Q}(\sqrt{3}))\right)=$ $\operatorname{rank}\left(E_{1, \theta}(\mathbf{Q})\right)+\operatorname{rank}\left(E_{3, \theta}(\mathbf{Q})\right)=0$.

The following theorem classifies all types of $(\mathbf{K}, \theta, n)$-triangles.

THEOREM 1.2. Assume that $n$ is not a $\theta$-congruent number over $\mathbf{Q}$ and let $\sigma$ be the generator of $\mathrm{Gal}(\mathbf{K} / \mathbf{Q})$. Then any $(\mathbf{K}, \theta, n)$-triangle with $(U, V, W) \in\left(\mathbf{K}^{*}\right)^{3}$ and $(0<U \leq V<W)$ is necessarily one of the following types:

Type 1. $U \sqrt{m}, V \sqrt{m}, W \sqrt{m} \in \mathbf{Q}$

Type 2. $U, V, W \sqrt{m} \in \mathbf{Q}$;

Type 3. $U, V \in \mathbf{K} \backslash \mathbf{Q}$ such that $\sigma(U)=V, W \in \mathbf{Q}$;

Type 4. $U, V \in \mathbf{K} \backslash \mathbf{Q}$ such that $\sigma(U)=-V, W \in \mathbf{Q}$.

Let $A=\operatorname{sqf}\left(r^{2}-s^{2}\right), B=\operatorname{sqf}(2 r(r-s))$ and $C=\operatorname{sqf}(2 r(r+s))$. The following proposition shows when there is no $(\mathbf{K}, \theta, n)$-triangle of Types 2,3 and 4 .

Proposition 1.3. Let $p$ be a prime number and the pair $(m, A)$ (resp. $(m, B)$ and $(m, C))$ can be written as $\left(p^{\alpha} a, p^{\beta} b\right)$, where $\alpha, \beta \in\{0,1\}$ and $\operatorname{gcd}(p, a b)=1$. Then there is no $(\mathbf{K}, \theta, n)$-triangle of Type 2 (resp. Type 3 and Type 4) whenever one of the following conditions hold. 
(1) $\underline{p=2:}(\alpha, \beta)=(0,0)$ and $(a, b) \stackrel{4}{\equiv}(3,3)$, $\overline{(\alpha, \beta)}=(0,1)$ and $(a, b) \stackrel{8}{\equiv}(3,1),(3,5),(7,5),(7,7)$, $(\alpha, \beta)=(1,0) \quad$ and $\quad(a, b) \stackrel{8}{=}(1,3),(1,5),(3,5),(3,7),(5,3),(5,7),(7,3)$, $(7,7)$, $(\alpha, \beta)=(1,1) \quad$ and $\quad(a, b) \stackrel{8}{\equiv}(1,3),(1,5),(3,1),(3,3),(5,1),(5,7),(7,5)$, $(7,7)$;

(2) $\underline{p \stackrel{4}{=} 1:}(\alpha, \beta)=(0,1)$ and $\left(\frac{a}{p}\right)=-1,(\alpha, \beta)=(1,0)$ and $\left(\frac{b}{p}\right)=-1$, $(\alpha, \beta)=(1,1)$ and $\left(\frac{a}{p}\right)\left(\frac{b}{p}\right)=-1 ;$

(3) $\underline{p \stackrel{4}{\equiv} 3:}(\alpha, \beta)=(0,1)$ and $\left(\frac{a}{p}\right)=-1,(\alpha, \beta)=(1,0)$ and $\left(\frac{b}{p}\right)=-1$, $(\alpha, \beta)=(1,1)$ and $\left(\frac{a}{p}\right)\left(\frac{b}{p}\right)=1$.

The next result settles a condition on $n$ and $m n$ to be $\theta$-congruent over $\mathbf{Q}$.

THEOREM 1.4. Let $n$ be a positive squarefree integer such that $\operatorname{gcd}(m, n)=1$ and $m n \neq 2,3,6$. Then the following statements are equivalent.

(1) There is a $(\mathbf{K}, \theta, n)$-triangle $(U, V, W)_{\theta}$ with $0<U \leq V<W, W \notin \mathbf{Q}$ and $W \sqrt{m} \notin \mathbf{Q}$;

(2) The integers $n$ and $m n$ are $\theta$-congruent numbers over $\mathbf{Q}$.

\section{Preliminaries}

Consider an elliptic curve $E: y^{2}=x^{3}+a x^{2}+b x+c$ over $\mathbf{Q}$. Recall that the $m$-twist $E^{m}$ of $E$ is an elliptic curve over $\mathbf{Q}$ defined by $y^{2}=x^{3}+a m x^{2}+$ $b m^{2} x+c m^{3}$. The next result establishes a fact about ranks [10].

THEOREM 2.1. Let $E$ be an elliptic curve over $\mathbf{Q}$. Then

$$
\operatorname{rank}(E(\mathbf{K}))=\operatorname{rank}(E(\mathbf{Q}))+\operatorname{rank}\left(E^{m}(\mathbf{Q})\right) .
$$

We denote the torsion subgroup of the groups $E(\mathbf{K})$ and $E^{m}(\mathbf{K})$ by $T(E, \mathbf{K})$ and $T\left(E^{m}, \mathbf{K}\right)$, respectively. Also, we write $T_{n, \theta}(\mathbf{K})$ and $T_{n, \theta}^{m}(\mathbf{K})$, respectively, in the case $E=E_{n, \theta}$. The following proposition and theorem have essential roles in the proof of our results.

Proposition 2.2 ([9, Proposition 1]). Let $E$ be an elliptic curve over $\mathbf{K}$. Then the map

$$
\phi: T(E, \mathbf{K}) / T(E, \mathbf{Q}) \rightarrow T\left(E^{m}, \mathbf{Q}\right), \quad \phi(\tilde{P}):=P-\sigma(P)
$$

is an injective map of abelian groups, where $\sigma$ is the generator of $\operatorname{Gal}(\mathbf{K} / \mathbf{Q})$. 
Theorem 2.3 ([7, Theorem 4.2]). Let $\mathbf{F}$ be an algebraic number field and $E$ an elliptic curve over $\mathbf{F}$ defined by

$$
y^{2}=\left(x-\alpha_{1}\right)\left(x-\alpha_{2}\right)\left(x-\alpha_{3}\right), \quad \alpha_{1}, \alpha_{2}, \alpha_{3} \in \mathbf{F} .
$$

Suppose that $\left(x_{0}, y_{0}\right)$ be an $\mathbf{F}$-rational point of $E$. Then, there exists an $\mathbf{F}$-rational point $\left(x_{1}, y_{1}\right)$ with $2\left(x_{1}, y_{1}\right)=\left(x_{0}, y_{0}\right)$ if and only if $x_{0}-\alpha_{1}, x_{0}-\alpha_{2}, x_{0}-\alpha_{3}$ are squares in $\mathbf{F}$. over $\mathbf{Q}$.

The next results give important information about $\theta$-congruent numbers

Theorem 2.4 (Fujiwara, [4]). Consider $0<\theta<\pi$ with rational cosine.

(1) A positive integer $n$ is a $\theta$-congruent number if and only if $E_{n, \theta}(\mathbf{Q})$ has a point of order greater than 2 ;

(2) If $n \neq 1,2,3,6$, then $n$ is a $\theta$-congruent number if and only if $E_{n, \theta}(\mathbf{Q})$ has positive rank.

All possibilities for the torsion subgroup of $E_{n, \theta}(\mathbf{Q})$ can be found in the next result.

TheOREM 2.5 (Fujiwara, [5]). Let $T_{n, \theta}(\mathbf{Q})$ be the torsion subgroup of the $\theta$-congruent number elliptic curve $E_{n, \theta}$ over $\mathbf{Q}$.

(1) $T_{n, \theta}(\mathbf{Q}) \cong \mathbf{Z}_{2} \oplus \mathbf{Z}_{8}$ if and only if there exist integers $a, b>0$ such that $\operatorname{gcd}(a, b)=1, a$ and $b$ have opposite parity and satisfy either of the following conditions.

(i) $n=1, r=8 a^{4} b^{4}, r-s=(a-b)^{4},(1+\sqrt{2}) b>a>b$,

(ii) $n=2, r=\left(a^{2}-b^{2}\right)^{4}, r-s=32 a^{4} b^{4}, a>(1+\sqrt{2}) b$;

(2) $T_{n, \theta}(\mathbf{Q}) \cong \mathbf{Z}_{2} \oplus \mathbf{Z}_{6}$ if and only if there exist integers $u, v>0$ such that $\operatorname{gcd}(u, v)=1, u>2 v$ and satisfy one of the following conditions:

(i) $n=1, r=\frac{1}{2}(u-v)^{3}(u+v), r+s=u^{3}(u-2 v)$,

(ii) $n=2, r=(u-v)^{3}(u+v), r+s=2 u^{3}(u-2 v)$,

(iii) $n=3, r=\frac{1}{6}(u-v)^{3}(u+v), r+s=\frac{1}{3} u^{3}(u-2 v)$,

(iv) $n=6, r=\frac{1}{3}(u-v)^{3}(u+v), r+s=\frac{2}{3} u^{3}(u-2 v)$;

(3) $T_{n, \theta}(\mathbf{Q}) \cong \mathbf{Z}_{2} \oplus \mathbf{Z}_{4}$ if and only if either of the following holds.

(i) $n=1,2 r$ and $r-s$ are squares but not satisfy (i) of Part (1),

(ii) $n=2, r$ and $2(r-s)$ are squares but not satisfy (ii) of Part (1);

(4) Otherwise, $T_{n, \theta}(\mathbf{Q}) \cong \mathbf{Z}_{2} \oplus \mathbf{Z}_{2}$.

Remark 2.6. For any squarefree integer $m>1$, the $m$-twist $E_{n, \theta}^{m}$ of the elliptic curve $E_{n, \theta}$ is defined by $y^{2}=x(x+(r+s) m n)(x-(r-s) m n)$, which is equal to $E_{m n, \theta}$, as seen. Therefore $E_{n, \theta}^{m}(\mathbf{Q})=E_{m n, \theta}(\mathbf{Q})$, and hence $T_{n, \theta}^{m}(\mathbf{Q})=$ $T_{m n, \theta}(\mathbf{Q})$. 


\section{Proofs}

Appealing to Proposition 2.2, we first settle all possibilities for the torsion subgroup of $E_{n, \theta}(\mathbf{K})$. Let $h, k$, and $d$ be integers such that $2 r=h^{2} \operatorname{sqf}(2 r)$, $r-s=k^{2} \operatorname{sqf}(r-s)$ and $2 r(r-s)=d^{2} m$, where $m=\operatorname{sqf}(2 r(r-s))$.

Proposition 3.1. Assume that $m>1$ and $n$ are squarefree positive integers such that $\operatorname{gcd}(m, n)=1$ and $m n \neq 2,3,6$. Let $T_{n, \theta}(\mathbf{K})$ be the torsion subgroup of $E_{n, \theta}(\mathbf{K})$.

(1) If $m=\operatorname{sqf}(2 r(r-s))$ and $n=\operatorname{sqf}(2 r)$, then

$$
\begin{aligned}
& T_{n, \theta}(\mathbf{K})=\{\infty,(0,0),(-(r+s) n, 0),((r-s) n, 0), \\
&\left((n h)^{2}-n d \sqrt{m}, \pm\left(\frac{d^{2} m n}{h}-n^{2} h d \sqrt{m}\right)\right), \\
&\left.\left((n h)^{2}+n d \sqrt{m}, \pm\left(\frac{d^{2} m n}{h}+n^{2} h d \sqrt{m}\right)\right)\right\} ;
\end{aligned}
$$

(2) If $m=\operatorname{sqf}(2 r(r-s))$ and $n=\operatorname{sqf}(r-s)$, then

$$
\begin{aligned}
& T_{n, \theta}(\mathbf{K})=\{\infty,(0,0),(-(r+s) n, 0),((r-s) n, 0), \\
&\left((n k)^{2}-n d \sqrt{m}, \pm\left(\frac{d^{2} m n}{k}-n^{2} k d \sqrt{m}\right)\right), \\
&\left.\left((n k)^{2}+n d \sqrt{m}, \pm\left(\frac{d^{2} m n}{k}+n^{2} k d \sqrt{m}\right)\right)\right\} ;
\end{aligned}
$$

(3) Otherwise, $T_{n, \theta}(\mathbf{K})=\{\infty,(0,0),(-(r+s) n, 0),((r-s) n, 0)\}$.

Proof. The 2-torsion subgroup of $E_{n, \theta}(\mathbf{K})$ is:

$$
E_{n, \theta}[2](\mathbf{K})=\{\infty,(0,0),(-(r+s) n, 0),((r-s) n, 0)\} .
$$

Therefore, we have $T_{n, \theta}(\mathbf{K}) \supset E_{n, \theta}[2](\mathbf{K}) \cong \mathbf{Z} / 2 \mathbf{Z} \oplus \mathbf{Z} / 2 \mathbf{Z}$. By Remark 2.6 and Theorem 2.5, $\quad T_{n, \theta}^{m}(\mathbf{Q})=T_{m n, \theta}(\mathbf{Q}) \cong \mathbf{Z} / 2 \mathbf{Z} \oplus \mathbf{Z} / 2 \mathbf{Z}$. Since $T_{n, \theta}(\mathbf{Q}) \cong \mathbf{Z} / 2 \mathbf{Z} \oplus$ $\mathbf{Z} / 2 \mathbf{Z}$, by Proposition 2.2 and [9, Theorem 1] we have

$$
T_{n, \theta}(\mathbf{K}) \cong \mathbf{Z} / 2 \mathbf{Z} \oplus \mathbf{Z} / 2 \mathbf{Z} \text { or } \mathbf{Z} / 2 \mathbf{Z} \oplus \mathbf{Z} / 4 \mathbf{Z} \text {. }
$$

First let $T_{n, \theta}(\mathbf{K}) \cong \mathbf{Z} / 2 \mathbf{Z} \oplus \mathbf{Z} / 4 \mathbf{Z}$. Then there exists a point $P=\left(x_{0}, y_{0}\right)$ of order 4 in $T_{n, \theta}(\mathbf{K})$. Then $2 P$ must be one of the points $(0,0),(-(r+s) n, 0)$ and $((r-s) n, 0)$. If $2 P=(0,0)$ then both $(r+s) n$ and $-(r-s) n$ are squares in $\mathbf{K}$, which is impossible since $\mathbf{K}$ is a real quadratic number field and hence -1 is not a square in $\mathbf{K}$. Similarly, if $2 P=(-(r+s) n, 0)$, then $-(r+s) n$ and $-2 r n$ are squares in $\mathbf{K}$, again a contradiction by the same reason. If $2 P=((r-s) n, 0)$, 
then $(r-s) n$ and $2 r n$ are squares in $\mathbf{K}$. Since $n$ is squarefree, these integers are squares in $\mathbf{K}$ if $m=\operatorname{sqf}(2 r(r-s))$. By a simple computation using the duplication formula we obtain (1) and (2). Now, $T_{n, \theta}(\mathbf{K}) \cong \mathbf{Z} / 2 \mathbf{Z} \oplus \mathbf{Z} / 2 \mathbf{Z}$ implies (3), and the proof is completed.

Proof of Theorem 1.1. Consider the two sets

$$
\begin{gathered}
S=\left\{(U, V, W) \in\left(\mathbf{K}^{*}\right)^{3}: 0<U \leq V<W, U V=2 r n\right. \text { and } \\
\left.U^{2}+V^{2}-2 s U V / r=W^{2}\right\}, \\
T=\left\{(u, v) \in 2 E_{n, \theta}(\mathbf{K}) \backslash\{\infty\}: v \geq 0\right\} .
\end{gathered}
$$

There is a one to one correspondence between the two sets $S$ and $T$ via the two mutually inverse maps $\varphi: S \rightarrow T$ and $\psi: T \rightarrow S$ defined by

$$
\begin{gathered}
\varphi(U, V, W):=\left(W^{2} / 4, W\left(V^{2}-U^{2}\right) / 8\right), \\
\psi(u, v):=(\sqrt{u+(r+s) n}-\sqrt{u-(r-s) n}, \sqrt{u+(r+s) n}+\sqrt{u-(r-s) n}, 2 \sqrt{u}) .
\end{gathered}
$$

Clearly, $E_{n, \theta}(\mathbf{K}) \backslash E_{n, \theta}[2](\mathbf{K}) \neq \emptyset$ if and only if $S \neq \emptyset$.

Suppose that $m \neq \operatorname{sqf}(2 r(r-s))$ and $m n \neq 2,3,6$. Then by proposition 3.1, we have $T_{n, \theta}(\mathbf{K})=E_{n, \theta}[2](\mathbf{K})$. Therefore, $\operatorname{rank}\left(E_{n, \theta}(\mathbf{K})\right)>0$ if and only if $E_{n, \theta}(\mathbf{K}) \backslash E_{n, \theta}[2](\mathbf{K}) \neq \emptyset$. So $\operatorname{rank}\left(E_{n, \theta}(\mathbf{K})\right)>0$ if and only if either $\operatorname{rank}\left(E_{n, \theta}(\mathbf{Q})\right)>0$ or $\operatorname{rank}\left(E_{n, \theta}^{m}(\mathbf{Q})\right)>0$, by Theorem 2.1. The second part of the theorem follows from Remark 2.6.

Proof of Theorem 1.2. Assume $n$ is a $(\mathbf{K}, \theta)$-congruent number and $(U, V, W)_{\theta}$ is the corresponding $(\mathbf{K}, \theta, n)$-triangle with area $n \alpha_{\theta}$ such that $0<$ $U \leq V<W$. As in the proof of the Theorem 1.1, there is a point $P=(x, y)$ in $E_{n, \theta}(\mathbf{K}) \backslash E_{n, \theta}[2](\mathbf{K})$ such that $\psi(P)=(U, V, W)$. Substituting $P$ by $P+(0,0)$, $P+(-(r+s) n, 0)$ or $P+((r-s) n, 0)$, if necessary, we may assume that $x>$ $[(r+s)+\sqrt{2 r(r-s)}] n$. Putting $2 P=(u, v)$ and using the map $\psi$ in the proof of Theorem 1.1, we obtain

$$
U=2 r n x /|y|, \quad V=x^{2}+2 \operatorname{sn} x-\left(r^{2}-s^{2}\right) n^{2} /|y|, \quad W=x^{2}+\left(r^{2}-s^{2}\right) n^{2} /|y|,
$$

where $x, y \in \mathbf{K}$ and $|\cdot|$ is the usual absolute value induced from the embedding $\imath: \mathbf{K} \hookrightarrow \mathbf{R}$ with $l(\sqrt{m})$ positive. Suppose $\sigma$ is a generator of $\operatorname{Gal}(\mathbf{K} / \mathbf{Q})$ and put $\sigma(P)=(\sigma(x), \sigma(y))$. Since $P+\sigma(P)$ is an element of $E_{n, \theta}(\mathbf{Q})$ and $n$ is not a $\theta$-congruent number, $P+\sigma(P) \in T_{n, \theta}(\mathbf{Q})=\{\infty,(0,0),(-(r+s) n, 0),((r-s) n, 0)\}$. Hence, one of the following cases necessarily happens:

I. $P+\sigma(P)=\infty$. In this case, $\sigma(x)=x$ and $\sigma(y)=-y$. So, $x, y \sqrt{m}$ and hence $U \sqrt{m}, V \sqrt{m}$ and $W \sqrt{m}$ are rational and we obtain a $(\mathbf{K}, \theta, n)$-triangle of Type 1 .

II. $P+\sigma(P)=(0,0)$. We have $\sigma(x) / x=\sigma(y) / y$, which we denote by $\alpha$. Then,

$$
\sigma(y)^{2}=\alpha^{2} y^{2}=\alpha^{2} x^{3}+2 \operatorname{sn} \alpha^{2} x^{2}-\left(r^{2}-s^{2}\right) n^{2} \alpha^{2} x .
$$


Since $\sigma(P)$ is a point on $E_{n, \theta}$, we get

$$
\begin{aligned}
\sigma(y)^{2} & =\sigma(x)^{3}+2 \operatorname{sn} \sigma(x)^{2}-\left(r^{2}-s^{2}\right) n^{2} \sigma(x) \\
& =\alpha^{3} x^{3}+2 \operatorname{sn} \alpha^{2} x^{2}-\left(r^{2}-s^{2}\right) n^{2} \alpha x .
\end{aligned}
$$

Clearly, $\alpha \neq 0,1$ and $x \neq 0$, which implies $x \sigma(x)=\alpha x^{2}=-\left(r^{2}-s^{2}\right) n^{2}$. Therefore,

$$
V=x(x+2 s n+\sigma(x)) /|y|, \quad W \sqrt{m}=x(x-\sigma(x)) \sqrt{m} /|y| .
$$

Since $x / y=\sigma(x / y)$ and $x>[(r+s)+\sqrt{2 r(r-s)}] n$, then $x /|y|$ is rational and hence $U=2 r n x /|y|, V$ and $W \sqrt{m}$ are rational, which gives a $(\mathbf{K}, \theta, n)$-triangle of Type 2 .

III. $P+\sigma(P)=((r-s) n, 0)$. We have $\sigma(x-(r-s) n) /(x-(r-s) n)=$

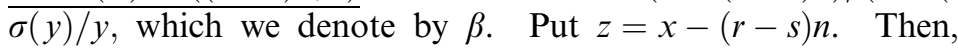

$$
\sigma(y)^{2}=\beta^{2}\left[z^{3}+(3 r-s) n z^{2}+2 r(r-s) n^{2} z\right] .
$$

Since $\sigma(P)$ is a point on $E_{n, \theta}$, we get

$$
\sigma(y)^{2}=\beta^{3} z^{3}+(3 r-s) n \beta^{2} z^{2}+2 r(r-s) n^{2} \beta z .
$$

Now $\beta \neq 0,1$ and $z \neq 0$, which implies $\beta z^{2}=2 r(r-s) n^{2}$. Substituting this equation and $x=z+(r-s) n$ in $U, V$ and $W$, we obtain

$$
U=\frac{z(\sigma(z)+2 r n)}{|y|}, \quad V=\frac{z(z+2 r n)}{|y|}, \quad W=\frac{z(z+2(r-s) n+\sigma(z))}{|y|} .
$$

Since $z / y=\sigma(z / y)$ and $z>0$, then $z /|y|$ and hence $W$ is rational and $\sigma(U)=V$. This time we obtain a $(\mathbf{K}, \theta, n)$-triangle of Type 3 .

IV. $\frac{P+\sigma(P)=(-(r+s) n, 0)}{\text { and }}$. Put $w=x+(r+s) n$. As in Case III, $w /|y|$

$$
W=w(w-2(r+s) n+\sigma(w)) /|y|
$$

are rational and $\sigma(U)=-V$, where

$$
U=w(2 r n-\sigma(w)) /|y|, \quad V=w(w-2 r n) /|y| .
$$

Therefore, we obtain a $(\mathbf{K}, \theta, n)$-triangle of Type 4 .

Proof of Proposition 1.3. If we suppose that there is a $(\mathbf{K}, \theta, n)$-triangle of Type 2, say $(U, V, W)_{\theta}=(u, v, w \sqrt{m})$ with $u, v, w \in \mathbf{Q}^{+}$, then $(x, y, z)=$ $(r u-s v, v, m r w)$ is a non-zero solution of the equation

$$
z^{2}=m x^{2}+m\left(r^{2}-s^{2}\right) y^{2} .
$$


And, if there is a $(\mathbf{K}, \theta, n)$-triangle of Type 3, say $(U, V, W)_{\theta}=(u-v \sqrt{m}$, $u+v \sqrt{m}, w)$ such that $\sigma(U)=V$, then $(x, y, z)=(u, v, r w)$ is a non-zero solution of

$$
z^{2}=2 r(r-s) x^{2}+2 m r(r+s) y^{2} .
$$

Similarly, if $(U, V, W)_{\theta}=(-u+v \sqrt{m}, u+v \sqrt{m}, w)$ is a $(\mathbf{K}, \theta, n)$-triangle of Type 4 such that $\sigma(U)=-V$, then $(x, y, z)=(u, v, r w)$ satisfies

$$
z^{2}=2 r(r+s) x^{2}+2 m r(r-s) y^{2} .
$$

By the Hasse local-global principle, the equations (3.1), (3.2) and (3.3) have solutions in $\mathbf{Q}$ if and only if they have a solution in $\mathbf{Q}_{p}$ for every prime $p$, where $\mathbf{Q}_{p}$ is the field of $p$-adic numbers. We assume that $A=\operatorname{sqf}\left(r^{2}-s^{2}\right)$, and for a prime $p$ the pair $(m, A)((m, B)$, and $(m, C)$, resp.) can be written as $\left(p^{\alpha} a, p^{\beta} b\right)$, where $\alpha, \beta \in\{0,1\}$ and $\operatorname{gcd}(p, a, b)=1$. Then, using Hilbert symbols [11, Theorem 1, III], the equations (3.1), (3.2) and (3.3) have solutions in $\mathbf{Q}_{2}$ if and only if one of the following cases happens:

i) $(\alpha, \beta)=(0,0)$ and $(a, b) \underset{8}{\not}(3,3)$;

ii) $(\alpha, \beta)=(0,1)$ and $(a, b) \stackrel{8}{\not} \underset{8}{(3,1)},(3,5),(7,5),(7,7)$;

iii) $(\alpha, \beta)=(1,0)$ and $(a, b) \stackrel{8}{\not \equiv}(1,3),(1,5),(3,5),(3,7),(5,3),(5,7),(7,3)$, $(7,7)$

iv) $(\alpha, \beta)=(1,1)$ and $(a, b) \stackrel{8}{\not \equiv}(1,3),(1,5),(3,1),(3,3),(5,1),(5,7),(7,5)$, $(7,7)$.

Also, the equations (3.1), (3.2) and (3.3) have solutions in $\mathbf{Q}_{p}$ with $p \stackrel{4}{=} 1$ if and only if one of the following happens:

i) $(\alpha, \beta)=(0,1)$ and $\left(\frac{a}{p}\right)=1$;

ii) $(\alpha, \beta)=(1,0)$ and $\left(\frac{b}{p}\right)=1$;

iii) $(\alpha, \beta)=(1,1)$ and $\left(\frac{a}{p}\right)\left(\frac{b}{p}\right)=1$.

Proof of Theorem 1.4. CASE $1 . \quad n$ and $m n$ are $(\mathbf{Q}, \theta)$-congruent numbers. Consider the $(\mathbf{Q}, \theta, n)$-triangle $\left(U_{1}, V_{1}, W_{1}\right)_{\theta}$ and the $(\mathbf{Q}, \theta, m n)$-triangle $\left(U_{2}, V_{2}, W_{2}\right)_{\theta}$, where

$$
\begin{aligned}
& 0<U_{1} \leq V_{1}<W_{1}, \quad 2 r n=U_{1} V_{1}, \quad U_{1}^{2}+V_{1}^{2}-\frac{2 s U_{1} V_{1}}{r}=W_{1}^{2}, \\
& 0<U_{2} \leq V_{2}<W_{2}, \quad 2 r m n=U_{2} V_{2}, \quad U_{2}^{2}+V_{2}^{2}-\frac{2 s U_{2} V_{2}}{r}=W_{2}^{2} .
\end{aligned}
$$

Hence, $\left(U_{2} / \sqrt{m}, V_{2} / \sqrt{m}, W_{2} / \sqrt{m}\right)_{\theta}$ is a $(\mathbf{K}, \theta, n)$-triangle. Recall the maps $\varphi$ and $\psi$ in the proof of Theorem 1.2 and put

$$
P=(u, v)=\varphi\left(\left(U_{1}, V_{1}, W_{1}\right)\right)+\varphi\left(\left(U_{2} / \sqrt{m}, V_{2} / \sqrt{m}, W_{2} / \sqrt{m}\right)\right) .
$$


Then the additive law on $E_{n, \theta}(\mathbf{K})$ implies $u=a+b \sqrt{m}$, where

$$
\begin{gathered}
a=\frac{m^{3} W_{1}^{2}\left(V_{1}^{2}-U_{1}^{2}\right)^{2}+W_{2}^{2}\left(V_{2}^{2}-U_{2}^{2}\right)^{2}}{4 m\left(W_{2}^{2}-m W_{1}^{2}\right)^{2}}-\left(\frac{W_{1}^{2}}{4}+\frac{W_{2}^{2}}{4 m}+2 s n\right)>0, \\
b=-\frac{W_{1} W_{2}\left(V_{1}^{2}-U_{1}^{2}\right)\left(V_{2}^{2}-U_{2}^{2}\right) \sqrt{m}}{2\left(W_{2}^{2}-m W_{1}^{2}\right)^{2}} .
\end{gathered}
$$

We may assume $v \geq 0$. Since $(u, v) \in T$, then $\psi((u, v)) \in S$ which indicates the sides of a $(\mathbf{K}, \theta, n)$-triangle $(U, V, W)_{\theta}$. In fact, if we suppose $U=u_{1}+u_{2} \sqrt{m}$, $V=v_{1}+v_{2} \sqrt{m}$ and $W=w_{1}+w_{2} \sqrt{m}$, where $u_{1}, u_{2}, v_{1}, v_{2}, w_{1}, w_{2}$ are rational, then

$$
w_{1}= \pm \sqrt{2\left(a \pm \sqrt{a^{2}-m b^{2}}\right)}, \quad w_{2}=\frac{2 b}{w_{1}}
$$

and

$$
U=\left(\alpha_{1}-\beta_{1}\right)+\left(\alpha_{2}-\beta_{2}\right) \sqrt{m}, \quad V=\left(\alpha_{1}+\beta_{1}\right)+\left(\alpha_{2}+\beta_{2}\right) \sqrt{m},
$$

where

$$
\begin{array}{ll}
\alpha_{1}= \pm \sqrt{\frac{(a+(r+s) n) \pm \sqrt{(a+(r+s) n)^{2}-m b^{2}}}{2}}, & \alpha_{2}=\frac{b}{2 \alpha_{1}} \\
\beta_{1}= \pm \sqrt{\frac{(a-(r-s) n) \pm \sqrt{(a-(r-s) n)^{2}-m b^{2}}}{2}}, & \beta_{2}=\frac{b}{2 \beta_{1}}
\end{array}
$$

Conversely, suppose to the contrary that $n$ or $m n$ is not $\theta$-congruent over $\mathbf{Q}$. First, assume $n$ is not $\theta$-congruent over $\mathbf{Q}$ but $m n$ is $\theta$-congruent over $\mathbf{Q}$. By Theorem $1.2(1)$, there is no $(\mathbf{K}, \theta, n)$-triangle $(U, V, W)_{\theta}$ satisfying the conditions $0<U \leq V<W, W \notin \mathbf{Q}$ and $W \sqrt{m} \notin \mathbf{Q}$.

CASE 2. $m n$ is not $\theta$-congruent over $\mathbf{Q}$ but $n$ is $(\mathbf{K}, \theta)$-congruent. Let $(U, V, W)_{\theta}$ denotes the sides of the corresponding $(\mathbf{K}, \theta, n)$-triangle. Multiplying the three sides by $\sqrt{m}$, we get the $(\mathbf{K}, \theta, m n)$-triangle $(U \sqrt{m}, V \sqrt{m}, W \sqrt{m})_{\theta}$. For the positive integer $m n$, we define the map $\varphi^{\prime}$ in the same way as $\varphi$. Put

$$
2 P^{\prime}=\varphi^{\prime}((U \sqrt{m}, V \sqrt{m}, W \sqrt{m}))
$$

for some point $P^{\prime} \in E_{m n, \theta}(\mathbf{K})$. For the generator $\sigma$ of $\operatorname{Gal}(\mathbf{K} / \mathbf{Q})$, since $P^{\prime}+\sigma\left(P^{\prime}\right)$ is an element in $E_{m n, \theta}(\mathbf{Q})$ and $m n$ is not $\theta$-congruent over $\mathbf{Q}$, we have

$$
P^{\prime}+\sigma\left(P^{\prime}\right) \in T_{m n, \theta}(\mathbf{Q})=\{\infty,(0,0),(-(r+s) m n, 0),((r-s) m n, 0)\} .
$$

Therefore, by the same way as in the proof of Theorem 1.2, one of the following cases necessarily happens: 
Type 1. $U, V, W \in \mathbf{Q}$

Type 2. $U \sqrt{m}, V \sqrt{m}, W \in \mathbf{Q}$;

Type 3. $U, V \in K \backslash \mathbf{Q}$ such that $\sigma(U)=V, W \sqrt{m} \in \mathbf{Q}$;

Type 4. $U, V \in K \backslash \mathbf{Q}$ such that $\sigma(U)=-V, W \sqrt{m} \in \mathbf{Q}$.

Hence, there is no $(\mathbf{K}, \theta, n)$-triangle $(U, V, W)_{\theta}$ with $W \notin \mathbf{Q}$ and $W \sqrt{m} \notin \mathbf{Q}$.

CASE 3. Both $n$ and $m n$ are not $\theta$-congruent numbers over $\mathbf{Q}$, where $m n \neq 2,3,6$. If $m \neq \operatorname{sqf}(2 r(r-s))$, by Theorem $1.1, n$ is not $(\mathbf{K}, \theta, n)$-congruent. If $m=\operatorname{sqf}(2 r(r-s))$ and $n$ is $(\mathbf{K}, \theta, n)$-congruent, we have $U=V$ for all $(\mathbf{K}, \theta, n)$ triangles $(U, V, W)_{\theta}$. Hence, there is no any $(\mathbf{K}, \theta, n)$-triangle $(U, V, W)_{\theta}$ with $W \notin \mathbf{Q}$ and $W \sqrt{m} \notin \mathbf{Q}$. We have completed the proof of Theorem 1.4.

\section{Examples}

In this section, we give some examples of $(\mathbf{K}, \theta)$-congruent numbers and verify all four types of $(\mathbf{K}, \theta, n)$-triangles in Theorem 1.2 in the cases $\theta=\pi / 3,2 \pi / 3$. Given $n$, let $(U, V, W)_{\theta}$ be a $(\mathbf{K}, \theta, n)$-triangle. Then, we have

$$
0<U \leq V<W, \quad U V=2 r n, \quad W^{2}=U^{2}+V^{2}-\frac{2 s}{r} U V .
$$

For any $(U, V, W)_{\theta}, \varphi((U, V, W))=\left(W^{2} / 4, W\left(V^{2}-U^{2}\right) / 8\right)$ is a point of $2 E_{n, \theta}(\mathbf{K}) \backslash\{\infty\}$. Also, for any point $(u, v) \in 2 E_{n, \theta}(\mathbf{K}) \backslash\{\infty\}$,

$$
\psi((u, v))=((\sqrt{u+(r+s) n}-\sqrt{u-(r-s) n}, \sqrt{u+(r+s) n}+\sqrt{u-(r-s) n}, 2 \sqrt{u})) .
$$

In our computations we have used Cremona's MWrank program [2] and the number theoretic Pari software [1].

I) Case $\theta=\pi / 3$. In this case, we have $r=2, s=1$, and $\alpha_{\theta}=\sqrt{3}$, and hence the area of any $(\mathbf{K}, \pi / 3, n)$-triangle is $n \sqrt{3}$.

Example 4.1. Take $n=3$ and $m=13$. We have the following $(\mathbf{Q}(\sqrt{13})$, $\pi / 3,3)$-triangles of types $1,2,3$ and 4 in Theorem 1.1 and the corresponding points in the set $2 E_{3, \pi / 3}(\mathbf{Q}(\sqrt{13})) \backslash\{\infty\}$.

Type 1. An easy computing shows that the rank of $E_{39, \pi / 3}(\mathbf{Q})$ is 2 , and the generators of the group are $P_{1}=[-9,-216]$ and $P_{2}=[75,-720]$. We have

$$
2 P_{1}=[1894 / 16,-91805 / 64] \in 2 E_{39, \theta}(\mathbf{Q}) \backslash\{\infty\} .
$$

Now, using the map $\varphi$ and $\psi$, defined in the proof of the Theorem 1.1 we get a rational $\pi / 3$-triangle $(13 / 2,24,43 / 2)$ with area 39 , which gives the following $(\mathbf{Q}(\sqrt{13}), \pi / 3,3)$-triangle of Type 1:

$$
(U, V, W)_{\pi / 3}=(\sqrt{13} / 2,24 \sqrt{13} / 13,43 \sqrt{13} / 26)
$$

which corresponds to the following point $Q=(1894 / 208$, $91805 \sqrt{13} / 416)$. 
Type 2. We have a $(\mathbf{Q}(\sqrt{13}), \pi / 3,3)$-triangle $(U, V, W)_{\pi / 3}=(3,4, \sqrt{13})$ of type 2 with the corresponding point $Q=(13 / 4,7 \sqrt{13} / 8)$.

Type 3. Let $U=u-v \sqrt{13}, \quad V=u+v \sqrt{13}$ and $W=w$, where $u, v, w \in$ $\mathbf{Q} \backslash\{0\}$. Then the pair $(u, v)$ satisfies the equation $u^{2}-13 v^{2}=12$. An easy solution of this equation is $\left(u_{0}, v_{0}\right)=(5,1)$. Parametrizing $u$ and $v$ in terms of $t \in \mathbf{Q}$ we obtain $u=-5 t^{2}+26 t-65 / t^{2}-13$ and $v=t^{2}-10 t+13 / t^{2}-13$. By putting these into $w^{2}=u^{2}+39 v^{2}$ and taking $t=13 / 4$ one can see that $w^{2}=u^{2}+39 v^{2}$ is a square in Q. So, we obtain $(U, V, W)_{\pi / 3}=(41-11 \sqrt{13} / 3,41+11 \sqrt{13} / 3$, $80 / 3)$, with $(\mathbf{Q}(\sqrt{13}), \pi / 3,3)$-triangle of type 3 with the corresponding point $Q=(1600 / 3,18040 \sqrt{13} / 9)$.

Type 4. Let $U=-u+v \sqrt{13}, \quad V=u+v \sqrt{13}$ and $W=w$, where $u, v, w \in$ $\mathbf{Q} \backslash\{0\}$. Then the pair $(u, v)$ satisfies $13 v^{2}-u^{2}=12$ with a solution $\left(u_{0}, v_{0}\right)=(1,1)$. A similar discussion as in the previous step, taking $t=8$, leads us to a $(\mathbf{Q}(\sqrt{13}), \pi / 3,3)$-triangle of Type 4 , with the corresponding point $Q=(24964 / 51,1002352 \sqrt{13} / 51)$.

Example 4.2. Let $n=11$ and $m=5$. One can see that $n$ is $\pi / 3$-congruent over $\mathbf{Q}$ and there is a $(\mathbf{Q}, \pi / 3,11)$-triangle $\left(U_{1}, V_{1}, W_{1}\right)=(55 / 12,48 / 5,499 / 60)$. Also, $n m=55$ is $\pi / 3$-congruent over $\mathbf{Q}$ and $\left(U_{2}, V_{2}, W_{2}\right)=(8,55 / 2,49 / 2)$ is a rational $\pi / 3$-triangle with area $11 \sqrt{3}$. Dividing its sides by $\sqrt{5}$, we obtain a $(\mathbf{Q}(\sqrt{5}), \pi / 3,11)$-triangle

$$
\left(U_{2} / \sqrt{5}, V_{2} / \sqrt{5}, W_{2} / \sqrt{5}\right)=(8 \sqrt{5} / 5,11 \sqrt{5} / 2,49 \sqrt{5} / 10)
$$

Now, a calculations as in the proof of Theorem 1.4 leads to a $(\mathbf{Q}(\sqrt{5}), \pi / 3,11)$ triangle

$$
\begin{aligned}
(U, V, W)=( & \frac{1}{310}(1470+499 \sqrt{5}), \frac{88}{5909}(1470-499 \sqrt{5}), \\
& \left.\frac{1}{183179}(4145193-12554399 \sqrt{5})\right)
\end{aligned}
$$

satisfying in Theorem 1.4.

II) Case $\theta=2 \pi / 3$. In this case, we have $r=2, s=-1$, and $\alpha_{\theta}=\sqrt{3}$. So, as in the case I, the area of any $(\mathbf{K}, 2 \pi / 3, n)$-triangle is $n \sqrt{3}$.

Example 4.3. Take $n=17$ and $m=13$. By a similar way as in Example 4.1, we find the following $(\mathbf{Q}(\sqrt{13}), 2 \pi / 3,17)$-triangles with area $17 \sqrt{13}$ of types $1,2,3$ and 4 preceding by their corresponding points in $2 E_{17,2 \pi / 3}(\mathbf{Q}(\sqrt{13})) \backslash\{\infty\}$.

Type 1. $(U, V, W)_{2 \pi / 3}=(17 \sqrt{13} / 26,8 \sqrt{13}, 217 \sqrt{13} / 26), \quad Q=(47089 / 16$, $9325575 \sqrt{13} / 10816)$;

Type 2. $(U, V, W)_{2 \pi / 3}=(1,68,19 \sqrt{13}), Q=(13 / 4,7 \sqrt{13} / 8)$; 
Type 3. $(U, V, W)_{2 \pi / 3}=(9-\sqrt{13}, 9+\sqrt{13}, 16), Q=(64,72 \sqrt{13})$;

Type 4. $(U, V, W)_{2 \pi / 3}=(-5+7 \sqrt{13} / 3,5+7 \sqrt{13} / 3,44 / 3), \quad Q=(484 / 9$, $770 \sqrt{13} / 27)$.

Example 4.4. Let $n=19$ and $m=6$. Then 19 is a $2 \pi / 3$-congruent number over $\mathbf{Q}$ and there is a $(\mathbf{Q}, 2 \pi / 3,6)$-triangle $\left(U_{1}, V_{1}, W_{1}\right)=(544 / 105,1995 / 136$, $254659 / 14280)$ with area $19 \sqrt{3}$. Also, the integer $n m=114$ is a $2 \pi / 3$-congruent number over $\mathbf{Q}$ and $\left(U_{2}, V_{2}, W_{2}\right)=(5,912 / 10,469 / 5)$ is a $2 \pi / 3$-triangle with area $114 \sqrt{3}$ from which we obtain a $(\mathbf{Q}(\sqrt{6}), 2 \pi / 3,19)$-triangle

$$
(5 \sqrt{6} / 6,76 \sqrt{6} / 5,469 \sqrt{6} / 30) .
$$

By a similar methods as in Example 4.2, one can find a $(\mathbf{Q}(\sqrt{6}), 2 \pi / 3,19)$-triangle

$$
\begin{aligned}
(U, V, W)_{2 \pi / 3}=( & (25449816+4838521 \sqrt{6}) / 4683550 \\
& 20(4145193-12554399 \sqrt{6}) / 28499829, \\
& 7(3589965612532-2573211605723 \sqrt{6}) / 1170880474675),
\end{aligned}
$$

satisfying Theorem 1.4.

\section{REFERENCES}

[ 1 ] C. Batue, K. Belabas, D. Bernardi, H. Cohen and M. Oliver, The computer algebra system Pari/Gp, Universite Bordeaux I, 1999, http://pari.math.u-bordeaux.fr/.

[2] John Cremona, MWrank program for elliptic curves over Q, 2008, http://www.maths.nott. ac.uk/personal/jec/MWrank.

[ 3 ] A. S. Janfada, S. Salami, A. Dujella and C. J. Perel, On the high $\operatorname{rank} \pi / 3$ and $2 \pi / 3$ congruent number elliptic curves, Rocky Mountains Journal of Mathematics 44 (2014), 1867-1880.

[4] M. Fujiwara, $\theta$-congruent numbers, Number theory (K. Györy, A. Pethö and V. Sós, eds.), de Gruyter, 1997, 235-241.

[ 5 ] M. Fujiwara, Some properties of $\theta$-congruent numbers, Natur. Sci. Rep. Ochanomizu Univ. 52, no. 2 (2001), 1-8.

[6] M. KAN, $\theta$-congruent numbers and elliptic curves, Acta Arithmetica 94 (2000), 153-160.

[7] A. KNAPP, Elliptic curves, Princeton University Press, 1992.

[8] N. Koblitz, Introduction to elliptic curves and modular forms, Grad. texts in Math. 97, 2nd ed., Springer-Verlag, Berlin, 1993.

[9] S. Kwon, Torsion subgroups of elliptic curves over quadratic extensions, J. Number Theory 62 (1997), 144-162.

[10] P. SERF, The rank of elliptic curves over real quadratic number fields of class number 1 , Ph.D. Thesis, Universität des Saarlandes, Saarbrücken, 1995.

[11] J. P. SERre, A course in arithmetic, GTM 7, Springer-Verlag, 1973.

[12] J. H. Silverman, The arithmetic of elliptic curves, Grad. texts in Math. 106, 2nd ed., Springer-Verlag, 2009. 
[13] M. TADA, Congruent number over real quadratic fields, Hiroshima Math. J. 31 (2001), 331-343.

[14] S. YoshidA, Some variant of the congruent number problem, I, Kyushu J. Math. 55 (2001), 387-404.

\author{
Ali S. Janfada \\ DePartment of Mathematics \\ URMIA UNIVERSITY \\ URMIA \\ IRAN \\ E-mail: a.sjanfada@urmia.ac.ir \\ asjanfada@gmail.com \\ Sajad Salami \\ Instituto Da Mathematica E Estatistica \\ UERJ \\ BRAZIL \\ E-mail: sajad.salami@ime.uerj.br
}

